\title{
Response of a Loaded Electric Dipole in an Imperfectly Conducting Cylinder of Finite Length
}

\author{
Charles W. Harrison, Jr., ${ }^{2}$ and Ronold W. P. King ${ }^{3}$
}

(November 16, 1959)

\begin{abstract}
Analytical relationships are developed which permit calculation of the power in the load impedance of an electric probe, symmetrically located within an imperfectly conducting cylinder of small radius compared to the wavelength, in terms of the electric field incident upon the cylinder.
\end{abstract}

\section{Introduction}

The problem is to determine the power in the load impedance at the center of a short dipole receiving antenna that is located within an imperfectly conducting shield of cylindrical shape and finite length in terms of a known electric field outside the completely closed shield. It is assumed that the radius of the shield and length of the probe are small fractions of the wavelength. The solution consists of the successive determination of the following:

(a) The ratio of the electric field inside to the field on the outside surface of the shield.

(b) The ratio of the electric field on the outside surface of the hollow cylinder to the incident electric field.

(c) The effective length and the driving-point impedance of the center-loaded dipole inside the shield.

(d) The power in the load impedance of the dipole in terms of the electric field within the cylindrical shield.

\section{Ratio of the Electric Field Inside an Im- perfectly Conducting Shield to the Field on the Outside Surface $[1]^{4}$}

Figure 1 shows a tube with hemispherical end caps. Its inner radius is $a_{1}$, its outer radius $a_{2}$, and its axial length $2 l$. The metal annulus is region 1 , the spaces outside and inside the tube are regions 2 and 3 , respectively. Let the axis of the cylindrical shield coincide with the $z$ axis of a system of cylindrical coordinates $r, \theta$, and $z$. If the length of the tube is at least 10 times its diameter and $a_{2}<<\lambda$, it is a satisfactory approximation to assume the transverse distribution of the axial electric field at any cross section of the cylinder not too near its ends to be independent of its axial distribution. The field

\footnotetext{
1 This paper originally appeared as Sandia Corporation Technical Memorandum No. 457-58(14) dated December 5, 1958.

2 Member of the Scientific Staff of the Sandia Corporation, Sandia Base, Albuquerque, New Mexico.

3 Consultant to the Sandia Corporation and Gordon McKay Professor of Applied Physies, Harvard University.

4 Figures in brackets indicate the literature references at the end of this paper.
}

outside the cylinder is assumed to be directed parallel to its axis, i.e., in the $z$-direction.

The governing wave equation for the electric field $E=\hat{z} E_{z}$ in a homogeneous region with conductivity $\tilde{\sigma}_{1}>>\omega \epsilon_{0}$, dielectric constant $\epsilon_{1}=\epsilon_{0}$, and permeability $\mu_{1}$ is

$$
V^{2} \underset{\sim}{E}-j \omega \sigma_{1} \mu_{1} \underset{\sim}{E}=0 .
$$

A time dependence of the form $\exp (j \omega t)$ is assumed where $\omega=2 \pi f$ and $f$ is the frequency.

Owing to rotational symmetry and the assumed independence of the transverse and axial distributions, the equation for the transverse distribution of $E_{z}$ is

$$
\frac{\partial^{2} E_{z}(r)}{\partial r}+\frac{1}{r} \frac{\partial E_{z}(r)}{\partial r}+k_{m}^{2} E_{z}(r)=0,
$$

which has the general solution

$$
E_{z}(r)=A_{1} J_{0}\left(k_{m} r\right)+B_{1} N_{0}\left(k_{m} r\right) \quad a_{1} \leq r \leq a_{2},
$$

where

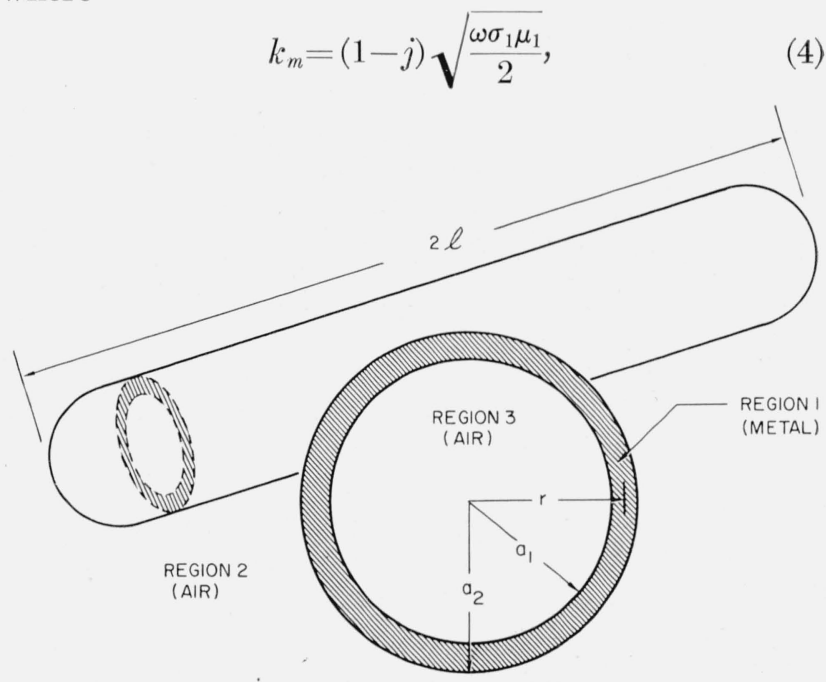

Figure 1. Cross section of hollow conductor. 
and $J_{0}(x)$ and $N_{0}(x)$ are Bessel functions of the first and second kind. In region 3 the free space wave equation,

$$
V^{2} \underset{\sim}{E}+\omega^{2} \mu_{0} \epsilon_{0} \underset{\sim}{E}=0
$$

applies; $\mu_{0}=4 \pi \times 10^{-7} \mathrm{~h} / \mathrm{m} ; \boldsymbol{\epsilon}_{0}=8.85 \times 10^{-12} \mathrm{f} / \mathrm{m}$.

The solution of (5) that remains finite at $r=0$ is

where

$$
E_{z}(r)=A_{3} J_{0}\left(k_{s} r\right) \quad 0 \leq r \leq a_{1},
$$

$$
k_{s}=\omega \sqrt{\mu_{0} \epsilon_{0}} .
$$

Now, if $\left(k_{s} a_{1}\right)^{2}<<1, J_{0}\left(k_{s} r\right) \approx 1$, and $E_{z}(r) \approx \mathrm{A}_{3}=$ const $\left(0 \leq r \leq a_{1}\right)$. It follows that within the cylindrical shell

$$
\frac{\partial E_{z}(r)}{\partial r}=0
$$

The Maxwell equation

$$
\operatorname{curl}_{\theta} \underset{\sim}{E}=-\frac{\partial E_{z}}{\partial r}=-j \omega B_{\theta}
$$

when combined with (8) leads to the conclusion that

$$
B_{\theta}(r)=0 \quad\left(0 \leq r \leq a_{1}\right) .
$$

Since the $B$-vector satisfies the boundary condition

$$
\mu_{0} B_{\theta 3}\left(a_{1}\right)=\mu_{1} B_{\theta_{1}}\left(a_{1}\right)
$$

at $r=a_{1}$, it follows with (8), (9), and (11) that

$$
j \omega B_{\theta_{1}}\left(a_{1}\right)=\left[\frac{\partial E_{z 1}(r)}{\partial r}\right]=0 \quad r=a_{1} .
$$

With (12), the constant $B_{1}$ may be evaluated in terms of $A_{1}$ from (3). The result is

$$
B_{1}=-A_{1} \frac{J_{1}\left(k_{m} a_{1}\right)}{N_{1}\left(k_{m} a_{1}\right)}
$$

The substitution of (13) in (3) and its evaluation successively at $r$ and $r=a_{2}$ leads directly to

$$
\begin{array}{r}
\frac{E_{z}(r)}{E_{z}\left(a_{2}\right)}=\frac{J_{0}\left(k_{m} r\right) N_{1}\left(k_{m} a_{1}\right)-N_{0}\left(k_{m} r\right) J_{1}\left(k_{m} a_{1}\right)}{J_{0}\left(k_{m} a_{2}\right) N_{1}\left(k_{m} a_{1}\right)-N_{0}\left(k_{m} a_{2}\right) J_{1}\left(k_{m} a_{1}\right)} \\
a_{1} \leq r \leq a_{2} .
\end{array}
$$

Finally, when $r=a_{1}$

$$
\frac{E_{z}\left(a_{1}\right)}{E_{z}\left(a_{2}\right)}=\frac{J_{0}\left(k_{m} a_{1}\right) N_{1}\left(k_{m} a_{1}\right)-N_{0}\left(k_{m} a_{1}\right) J_{1}\left(k_{m} a_{1}\right)}{J_{0}\left(k_{m} a_{2}\right) N_{1}\left(k_{m} a_{1}\right)-N_{0}\left(k_{m} a_{2}\right) J_{1}\left(k_{m} a_{1}\right)},
$$

which is the desired complex ratio of the field inside the imperfectly conducting hollow cylinder to the field on its outside surface.
In the important special case of large arguments defined by ${ }^{5}$

$$
a_{1} \sqrt{\omega \mu_{1} \sigma_{1}} \geq 10
$$

it is possible to use the well known asymptotic forms of the Bessel functions to simplify (15). The result is

$$
\frac{E_{z}\left(a_{1}\right)}{E_{z}\left(a_{2}\right)}=\sqrt{\frac{a_{2}}{a_{1}}}\left\{\frac{1}{\cos \left[k_{m}\left(a_{2}-a_{1}\right)\right]}\right\} \text {. }
$$

With (4) and standard formulas for the cosine with a complex argument, (17) becomes

$$
\frac{E_{z}\left(a_{1}\right)}{E_{z}\left(a_{2}\right)}=\sqrt{\frac{a_{2}}{a_{1}}}\left\{\frac{\cos \gamma \cosh \gamma-j \sin \gamma \sinh \gamma}{\cos ^{2} \gamma+\sinh ^{2} \gamma}\right\},
$$

and its magnitude is

$$
\frac{\left|E_{z}\left(a_{1}\right)\right|}{\left|E_{z}\left(a_{2}\right)\right|}=\sqrt{\frac{a_{2}}{a_{1}}}\left\{\frac{1}{\sqrt{\cos ^{2} \gamma+\sinh ^{2} \gamma}}\right\},
$$

where

$$
\gamma=\left(a_{2}-a_{1}\right) \sqrt{\frac{\omega \sigma_{1} \mu_{1}}{2}}
$$

Note that (18) and (19) are valid only when (16) is satisfied.

\section{Relation Between the Field Incident Upon an Imperfectly Conducting Hollow Cylin- der and the Field on its Outside Surface}

The volume density of current $i_{z}(r)$ in the conducting tube $\left(a_{1} \leq r \leq a_{2}\right)$ is related to the electric field $E_{z}(r)$ by the constitutive relation

$$
i_{z}(r)=\sigma_{1} E_{z}(r)
$$

It follows that

$$
\frac{i_{z}(r)}{i_{z}\left(a_{2}\right)}=\frac{E_{z}(r)}{E_{z}\left(a_{2}\right)}
$$

The total axial current $I(0)$ at the center $(z=0)$ of the cylinder is given by the formula

$$
I(0)=\int_{a_{1}}^{a}{ }_{2} i_{z}(r) 2 \pi r d r,
$$

where $i_{z}(r)$ is the transverse distribution of the current density across the center of the cylinder. The substitution of (22) with (14) in (23) and the subsequent integration gives the following expression for

${ }^{5}$ At $15 \mathrm{kc}$ an aluminum cylinder must be at least $0.447 \mathrm{~cm}$ in radius; an iron shield could be smaller. 
the current density near the outer surface in terms of the total current:

$$
\begin{aligned}
i_{z}\left(a_{2}\right) & =\frac{I(0)}{\pi a_{2}^{2}}\left(\frac{k_{m} a_{2}}{2}\right) \\
& \times\left\{\frac{J_{0}\left(k_{m} a_{2}\right) N_{1}\left(k_{m} a_{1}\right)-N_{0}\left(k_{m} a_{2}\right) J_{1}\left(k_{m} a_{1}\right)}{J_{1}\left(k_{m} a_{2}\right) N_{1}\left(k_{m} a_{1}\right)-N_{1}\left(k_{m} a_{2}\right) J_{1}\left(k_{m} a_{1}\right)}\right\} .
\end{aligned}
$$

The corresponding asymptotic formula for $a_{1} \sqrt{\omega \mu_{1} \sigma_{1}}$ $\geq 10$ is

$$
i_{z}\left(a_{2}\right)=\frac{I(0)}{\pi a_{2}^{2}}\left(\frac{k_{m} a_{2}}{2}\right) \cot \left[k_{m}\left(a_{2}-a_{1}\right)\right],
$$

so that with (21) at $r=a_{2}$

$$
E_{z}\left(a_{2}\right)=\frac{I(0)}{\pi \sigma_{1} a_{2}^{2}}\left(\frac{k_{m} a_{2}}{2}\right) \cot \left[k_{m}\left(a_{2}-a_{1}\right)\right] .
$$

This expression relates the electric field on the outside surface of the hollow cylinder halfway between its ends to the total current $I(0)$ traversing this cross section of the tube.

The axial distribution $I(z)$ of the current in the cylinder, in particular, its value $I(0)$ at the center, is known from antenna theory since the cylindrical shield is, in effect, a receiving antenna without load (refer to fig. 2). The current at its center is given by $[2]$

$$
I(0)=\frac{V_{\text {oc }}}{Z_{\text {in }}}=\frac{2 l_{e} E_{z}^{i}}{Z_{\text {in }}}
$$

where $V_{o c}$ is the open-circuit voltage given by the product of the effective length $2 l_{e}$ of the cylinder multiplied by the incident tangentially directed electric field $E_{z}^{i} . \quad Z_{i n}$ is the driving-point impedance of a symmetrical center-driven antenna with dimensions identical to those of the shield. The effective length $2 l_{e}$ of an electrically short antenna $\left(k_{s} l \leq 0.5\right)$ is given approximately by [3]

$$
2 l_{e} \approx l,
$$

where $l$ is the half-length of the shield and $k_{s}=2 \pi / \lambda$.

The impedance of a perfectly conducting short antenna [3] for which $k_{s} l \leq 0.5$ is given by the formula [4]

$$
\begin{aligned}
& Z_{0}^{r} \approx-j \frac{\eta}{2 \pi k_{s} l} \\
& \times\left\{(\Omega-2-2 \ln 2)\left(1-j \frac{\left(k_{s} l\right)^{3}}{3(\Omega-2-2 \ln 2)}\right)\right\} \text { ohms, }
\end{aligned}
$$

where

$$
\eta=\sqrt{\frac{\mu_{0}}{\epsilon_{0}}}=120 \pi
$$

and

$$
\Omega=2 \ln \left(\frac{2 l}{a_{2}}\right) \text {. }
$$

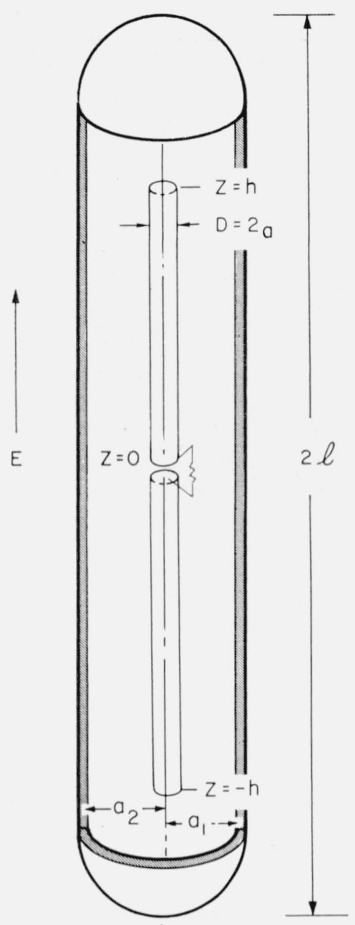

Figure 2. Electric probe symmetrically oriented along the axis of an imperfectly conducting cylinder.

The input impedance of the imperfectly conducting short dipole is approximately.

$$
Z_{\text {in }}=Z_{0}^{r}+R_{0}^{l}
$$

where $R_{0}^{l}$ is the ohmic loss resistance referred to the input terminals.

$R_{0}^{l}$ may be evaluated by noting that the current distribution along an electrically short antenna is approximately triangular in shape. Hence, the averagec urrent along the structure is $I_{a v}^{2}=\frac{1}{3} I^{2}(0)$. The ohmic resistance of the tube of length $2 l$ has been given by King [5]. It is

$$
R^{t}=\frac{l k_{m}}{\pi a_{2} \sigma_{1}}\left(\sqrt{\frac{\cosh 2 \gamma+\cos 2 \gamma}{\cosh 2 \gamma-\cos 2 \gamma}}\right) \cos \left(\psi_{r}-\psi_{c}+\frac{\pi}{4}\right),
$$

where

$$
\tan \psi_{r}=-\tanh \gamma \tan \gamma
$$

and

$$
\tan \psi_{c}=\frac{\tanh \gamma}{\tan \gamma}
$$

provided $a_{1} \sqrt{\omega \mu_{1} \sigma_{1}} \geq 10 . \gamma$ is defined by $(20)$. The power lost in heating the antenna is

$$
P_{L}=I^{2}(0) R_{0}^{l}=\left(\frac{I^{2}(0)}{3}\right) R^{t}
$$


Hence,

$$
R_{0}^{l}=\frac{R^{t}}{3}
$$

Accordingly,

$$
\begin{aligned}
& Z_{i n} \approx-j \frac{\eta}{2 \pi k_{s} l} \\
& \times\left\{(\Omega-2-2 \ln 2)\left(1-j \frac{\left(k_{s} l\right)^{3}}{3(\Omega-2-2 \ln 2)}\right)\right\}+\frac{R^{t}}{3} .
\end{aligned}
$$

The substitution of (27) in (26) gives

$$
E_{z}\left(a_{2}\right)=\frac{2 l_{e} E_{z}^{i}}{\pi Z_{i n} \sigma_{1} a_{2}^{2}}\left(\frac{k_{m} a_{2}}{2}\right) \cot \left[k_{m}\left(a_{2}-a_{1}\right)\right]
$$

Thus, if the incident field $E_{z}^{i}$ in the vicinity of the cylinder is known, (39) may be used to find the field $E_{z}\left(a_{2}\right)$ on the outside surface. The field within the cylinder may then be calculated from (18).

\section{Effective Height and Driving-Point Im- pedance of a Short Electric Dipole in an Imperfectly Conducting Cylinder}

To determine the behavior of a center-loaded electric dipole of half-length $h$ and radius $a$ symmetrically located along the axis of an imperfectly conducting cylinder requires not only a knowledge of the electric field inside the cylinder in terms of the incident electric field but also the effective length and driving-point impedance of the probe.

The effective length of a probe encased within an imperfectly conducting cylinder is the same as its effective length when isolated. The effective length is defined in terms of the field maintained along the probe by all currents other than those in the probe itself. This is precisely the field $E_{z}\left(a_{2}\right)$ maintained in the cylinder by the distant signal source and the current in the imperfectly conducting shield. (It is to be remembered that the field in the interior of the tube is uniform.) The only approximation involved is the assumption that the current in the probe does not react on currents in the shield to change them significantly. The required effective length $2 h_{e}$ may be calculated from (28) (or the formula in footnote 3 ) provided $h$ is written for $l$ throughout ( $h$ is the halflength of the electric probe).

The driving-point impedance of the electric probe encased in the imperfectly conducting cylinder lies between the impedance of an identical unshielded probe and the impedance of two imperfectly conducting sections of coaxial transmission-line in series. These sections are open-circuited, and have a length equal to the half-length $h$ of the probe. Capacitive end effects may be determined but are probably small enough to neglect. In terms of the electromagnetic field that would be set up by the probe antenna if it were used for transmission, three situations must be considered: (a) When the probe is isolated, i.e., is outside the metal cylinder, the distant field is due entirely to the currents in the antenna.

(b) When the probe is in a perfectly conducting cylinder, the field maintained at outside points by the currents in the antenna is exactly cancelled by the field set up by the currents in the shield.

(c) When the probe is in an imperfectly conducting cylinder, currents are induced in the shield which maintain a field that partially cancels the field of the probe, but this cancellation is not complete.

Thus the current in the probe can be separated into two parts. One part, $I_{L}$, is equal and opposite to the current in the shield; it is a transmission-line current. The other part, $I_{A}$, is the true antenna current. The total current $I_{t}$ in the unloaded probe is

$$
I_{t}=I_{A}+I_{L}
$$

The voltage driving the probe is

$$
V_{\mathrm{o}}=I_{t} Z_{0}=I_{A} Z_{A}+I_{L} Z_{L}=I_{t}\left\{Z_{L}-\frac{I_{A}}{I_{t}}\left(Z_{L}-Z_{A}\right)\right\}
$$

where $Z_{0}$ is the impedance of the probe when encased in the imperfectly conducting cylinder; $Z_{L}$ is the impedance of two perfectly conducting open-circuited sections of coaxial line that are of equal length and connected in series. $Z_{L}$ may be computed from the formula

$$
Z_{L}=-j 276 \log _{10}\left(\frac{a_{1}}{a}\right) \cot k_{s} h
$$

where $h$ is the half-length of the probe and $a$ is its radius; and $Z_{A}$ is the self-impedance of the probewhen isolated. It is given by (32) or by (29) when losses are negligible.

From (41), it follows that

$$
Z_{0}=\frac{V_{\mathrm{o}}}{I_{t}}=Z_{L}-\frac{I_{A}}{I_{t}}\left(Z_{L}-Z_{A}\right)
$$

The current $I_{A}$ is the current in the probe required to maintain the field $E_{z}\left(a_{2}\right)$ just outside the shield, if the shield were absent. $I_{t}$ is the current required to maintain the field $E_{2}^{i}\left(a_{1}\right)$ just inside the shield. Since the thickness of the shield is small compared with any radial distance over which $E_{z}$ could vary significantly in amplitude, it follows that

$$
\frac{I_{A}}{I_{t}} \sim \frac{E_{z}\left(a_{2}\right)}{E_{z}^{i}\left(a_{1}\right)}
$$

This complex ratio isappr oximately $E_{z}\left(a_{1}\right) / E_{z}^{i}$, obtained when (39) is combined with (17). The substitution of (44) in (43) gives

$$
Z_{0}=Z_{L}-\frac{E_{z}\left(a_{2}\right)}{E_{z}^{i}\left(a_{1}\right)}\left\{Z_{L}-Z_{A}\right\}
$$


This is the final formula for the driving-point impedance of an electric probe symmetrically situated within an imperfectly conducting cylinder.

It is important to observe that the ratio $E_{z}\left(a_{2}\right) /$ $E_{z}^{i}\left(a_{1}\right)$ in (45) is small, since when the probe is driven $E_{z}\left(a_{2}\right)$, the field on the outside of the shield is small, while the field on the inside of the shield, $E_{z}^{i}\left(a_{1}\right)$, is large. On the other hand in the receiving case $E_{z}^{i}$ is large and $E_{z}\left(a_{1}\right)$ is small, thus

$$
\left\{\frac{E_{z}\left(a_{2}\right)}{E_{z}\left(a_{1}\right)}\right\}=\left\{\frac{E_{z}\left(a_{1}\right)}{E_{z}\left(a_{2}\right)}\right\} .
$$

\section{Driven Probe Receiving Probe}

Since the ratio $E_{z}\left(a_{2}\right) / E_{z}^{i}\left(a_{1}\right)$ is small when the dipole is driven and the cylinder is a moderately good conductor, it follows that

$$
Z_{0} \approx Z_{L}
$$

On the other hand when the ratio $E_{z}\left(a_{2}\right) / E_{z}^{j}\left(a_{1}\right)$ $\rightarrow 1$, as when the cylindrical shield is nonexistent,

$$
Z_{0} \approx Z_{A}
$$

These relations for limiting values serve as checks on (45).

The equivalent receiving circuit of the probe consists of a generator with an emf equal to $2 h_{e} E_{z}\left(a_{1}\right)$ driving a circuit consisting of $Z_{0}$ in series with the load impedance. The power in the load is readily calculated in terms of the field $E_{z}\left(a_{1}\right)$. Thus

$$
P=\left|I^{2}(0)\right| R_{L} ; I(0)=\frac{2 h_{e} E_{z}\left(a_{1}\right)}{\left(R_{0}+R_{L}\right)+j\left(X_{0}+X_{L}\right)} .
$$

The impedance of an electric probe is essentially a capacitive reactance since the resistive component is only a fraction of an ohm. Maximum power is obtained in a given load when the probe is tuned to resonance by means of series inductors. If this is done, the full open-circuit, $V_{\text {oc }}$ appears across the load, if all losses are neglected. In this case $Z_{0}$ does not appear in the equivalent circuit of the probe, and (45) is not needed.

\section{Conclusion}

A method has been presented for calculating the power in the load of an electric probe encased in a partially conducting cylindrical shield. The analysis has been carried out for sinusoidal signals. If the signal source emits repetitive pulses, it is necessary to make a Fourier analysis of the incoming wave and solve the problem for the fundamental and several harmonic frequencies. The total power in the load impedance is then the sum of the powers absorbed at each frequency considered. Again it is assumed that the circuit dimensions are small in terms of the shortest wavelength of the signal component that contributes significantly to the power in the load.

The field incident upon the cylindrical shield need not be linearly polarized, as assumed in the analysis. An elliptically polarized electric field may be decomposed into two components which are fixed in space and which differ in phase, magnitude, and direction. In particular, the field may be resolved into two mutually perpendicular components which remain stationary in space. One of these components may be chosen parallel to the cylindrical shield, and the other perpendicular to it. Both vary periodically in time, but the latter contributes nothing to the induced voltage; its magnitude and phase are of no significance, and it may be ignored. Accordingly, maximum power is delivered to the load impedance of the probe when the hollow cylinder is oriented in space so that the major axis of the elliptical contour of the electric field is directed parallel to the axis of the cylindrical shield.

\section{References}

[1] A somewhat different analysis from that presented here for the field inside a conducting tube is given in R. W. P. King, Electromagnetic Engineering, pp. 350 to 359 (McGraw-Hill Book Co., Inc., New York, N.Y., 1945).

[2] R. W. P. King, Theory of Linear Antennas, Ch. IV,'Sec. 4 (Harvard University Press, 1956).

[3] More accurate formulas for the effective length and impedance of an electrically short antenna, specifically when $l / a_{2}=75$ are given by R. W. P. King, Theory of Linear Antennas, supra, p. 496. Specific formulas are $2 l_{e}=0.958 l\left\{1+0.061\left(k_{s} l\right)^{2}\right\} ; Z_{0}^{r}=18.3 k_{s}^{2} l^{2}\left(1+0.086 k_{s}^{2} l^{2}\right)$ $-j \frac{396}{k_{s} l}\left(1-0.383 k_{s}^{2} l^{2}\right)$ ohms.

[4] R. W. P. King, Theory of Linear Antennas, supra, p. 184, eq. (6a).

[5] R. W. P. King. Electromagnetic Engineering, supra, p. 357 , eq. (4).

\section{Supplementary References}

Louis V. King, Electromagnetic shielding at radio frequencies, Philosophical Magazine 15, 201 (Feb. 1933).

H. Kaden, Die elektromagnetische Schirmung in der Fernmelde und Hochfrequenztechnik (Springer-Verlag, 1950).

Boulder, Colo.

(Paper 64D3-60) 